\title{
DESIGN AND CONSTRUCTION OF A NEW GENERATION OF ROBOTS
}

\author{
Azam Salari Bajegani ${ }^{1}$ \\ Zahra Moeidi
}

\begin{abstract}
Each robot that is part of the physical environment, should be able to interact with humans. The dynamic balance on the robots such as the spherical robot cause demonstration Various interactive behaviors. Spherical robot is a stable and dynamic moving robot. It only features a spherical wheel that enables it to move in all directions, so there is not a problem to overturn the standing robots because its motion is in the form of rolling. The robot is able to easily move between the glades, sand

robot is more capable than other robots made with different movement mechanisms, so that it can be easily moved and smooth in smooth, fluid, slippery, and angular surfaces, without the need for an attachment to move, It just moves easily with the gravity of the earth. The propulsion mechanism of this robot is a pendulum of two degrees of freedom propelled by two engines. This robot is a non-holonomic and non-linear system, so it needs a nonlinear controller like Sliding Mode Control (SMC).
\end{abstract} and snow without clinging and can be used to build special operations teams in war zones or even carry explosives to the enemy's base. This research, is about the kinematics of the optimal motion of a spherical robot in a direct path with a simple mechanism and check spherical robot's control by the computer, and how wireless communication is communicated through the module. This
Keywords: spherical robot, automated control, non-holonomic system, pendulum, SMC.

\section{INTRODUCTION}

Robots were first used in the industry in 1954, that was a robot arm or a Manipulator that only had 3 degrees of

\footnotetext{
${ }^{1}$ Department of electrical engineering (control), Faculty of engineering, the University of Qom, Qom, Iran. Email: salari.azam90@gmail.com

${ }^{2}$ Department of electrical engineering (power system), Faculty of engineering, the Islamic Azad University of Kashan, Isfahan, Iran. Email: moeidi_z72@yahoo.com
} 
freedom. Today's industrial robots are often the same robotic arm, but with 6 degrees of freedom and much more advanced, they did not have to work in the past. Robots are used in the industry in many different ways and methods. In recent years, the use of mobile robots has been further developed to access larger workplaces and mobility capabilities. Hence, several mechanisms have been designed and developed to generate motion in robots. The use of wheel and legs is one of the driving mechanisms that has been studied for better production and maneuverability in various research centers. This issue is even more important when using mobile robots in space research and military activities. Spherical robot is a moving robot made up of a spherical shell and an internal stimulus mechanism. In this research, the spherical robot design and commissioning and wireless communication project will be investigated. For this purpose, the kinetic analysis of the robot is first performed and, in the end, the path is planned in different directions.

The use of robots in the work has many advantages that we will refer to later.

- Robots can do dangerous things like monitoring a nuclear power plant or exploring a volcano.

- Robots can do things more accurately than humans and accelerate the progress of medical science and other applied sciences.

- Robots are particularly useful in repetitive and tedious tasks, such as building circuit boards, pouring glue on spare parts, and more.

- Robotics and automation can in many cases increase safety, production, quality, and product quality.

- Robots can work in hazardous situations and thus save thousands of lives.

- Robots do not care about their surroundings and their human needs do not make sense. And the robots are never tired.

- The precision of the robots is much more than humans, they are at a millisecond or even micro-inch precision.

- Robots can do a few things at a moment, but humans do just one job at a moment.

\section{ROBOT AND ROBOTIC} ADVANTAGES 
- Robots are usually used in cases where they can do things better than humans or operate in high-risk environments.

All evaluations emphasize that robots will play an increasingly important role in modern societies. They continue to do dangerous, repetitive, costly and precise work to prevent people from doing so.

\section{ROBOTS}

\section{CATEGORIZATION}

Robots at different levels have two "performance variations" and "automatching capabilities with the environment." Based on these two features, the robots are categorized.

The division of the Japanese robot union (jira) is as follows:

1) A device that is controlled by the hand.

2) The robot for successive tasks unchanged
3) Robot for successive variable jobs

4) Embroidery Robot

5) Control Robot

6) Clever robot

In the category of the American Institute of Robotics (RIA), only 3 to 6 machine buses are considered robots.

The robot examined in this study can be classified as Category 1 or 5 .,

\section{THE IDEA OF THE PROJECT}

an amphibian robot that is built in American company, called GuardBot, is able to easily navigate through the glades, sand and snow. The creators of the robot believe that it can be used to build special operations teams in war zones or even carry explosives to the enemy base.

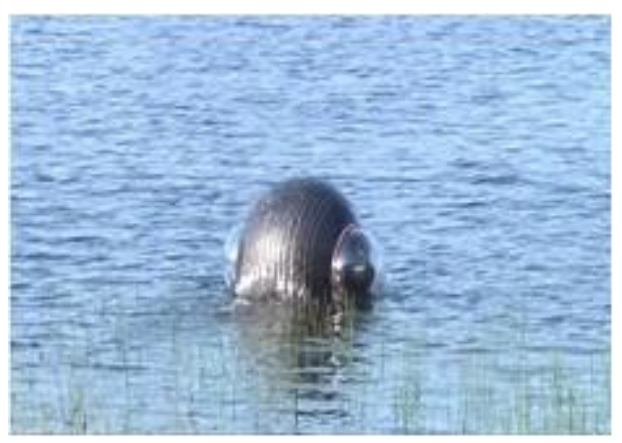




\section{Periódico do Núcleo de Estudos e Pesquisas sobre Gênero e Direito \\ Centro de Ciências Jurídicas - Universidade Federal da Paraíba V. 8 - No 02 - Ano 2019 - Special Edition ISSN | 2179-7137 | http://periodicos.ufpb.br/ojs2/index.php/ged/index}

Figure. 1. a view of an amphibian robot

The spherical design protects the robot, and sand and even dangerous gases can not enter and harm electronic devices. The cameras of the system provide 360-degree viewing angles, and the robot can carry bulk weights of up to two kilograms. The robot was originally designed for planetary missions, but was redesigned for use on Earth. The spherical robot is designed with the idea of this robot's structure.

\section{ECONOMICAL}

\section{JUSTIFICATION}

This robot is more capable than other robots made with different movement mechanisms, so that it can be easily moved and smooth in smooth, fluid, slippery, and angular surfaces, without the need for an attachment to move It just moves easily with the gravity of the earth. The same robot features and compatibility with all environments make it economically more economical than similar robots. The power consumption of this robot is low and there is little contact with the ground, which is also due to the cause.
Also, the friction in this robot is low and has a long lifespan and due to its spherical compartment, the parts are not exposed to environmental factors and therefore have a longer life span.

\section{CREATIVITY AND INNOVATION IN MANUFACTURING}

The robot is equipped with GPS and compass, and the robot can be automatically transferred from the current location on the map to the desired destination, as well as manual control through laptops, if necessary. The upper hemisphere of the robot is empty, so any other device can be added to the upper part, which has led the spider robot to be used for military affairs. Also, if artificial intelligence or an advanced algorithm, such as the neural network, is used in this robot, the learning power is given to the robot and, given that the horizontally located on the robot, always remains horizontal and constant, it can be used to design the robot. It can be used to process image processing by installing 
the camera on it and sending online images to the server computer.

\section{SPHERICAL MOTION MECHANISM}

\section{ROBOT}

The spherical robot actuator mechanism is designed to change the location of the robot's center of gravity by moving the pendulum in its center. The two main DC motors are used to move forward and backward with full freedom along the sphere diameter, and a servomotor adjuster pendulum with two degrees of freedom that changes the angular momentum of the spinning robot and moves to the sides. The spherical robot consists of three parts for moving mechanics, electronics for motion control and a computer for steering. We will continue to examine these components in full.

\subsection{The Mechanic}

The robot uses two gearboxes with speeds of 100 RPM to rotate the sphere and a servo motor (with a rotating angle control of 0 to 180 degrees) to move the pendulum inside the sphere.

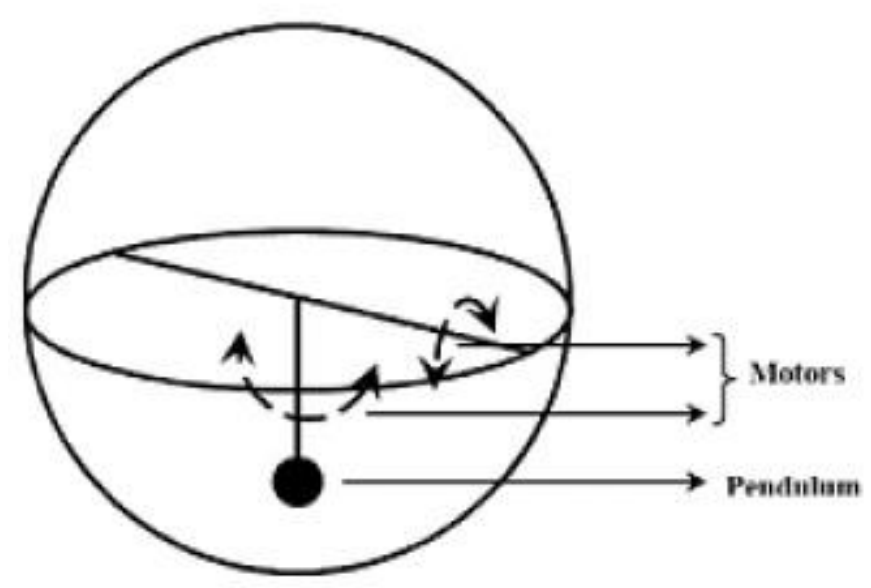

Figure. 2. Spherical robot components

The spherical robot uses its spherical shape to survive the movement and to support the movement of gravity. In this way, a clock-type pendulum fitted at the center of the robot and connected to a weight grabbed by ground force is the main point of the robot's spherical motion. The weight at the end of the 
pendulum is lower than the pendulum's pivot axis, resulting in a robot relying on its original axis. This is the case when motors start to rotate, due to lack of weight symmetry on the other side of the pendulum and the power not to rotate it within the crust of the robot, it begins to rotate and rotates the sphere of the pendulum path. The pendulum and other equipment in the shell of the sphere are fixed (without spin).

Pupose of moving to the pendulum path is that when the pendulum is in the middle, ie, completely perpendicular to the main axis of the robot's movement, the robot moves in the straight path. In this case, the pendulum has equally balanced weight for the sides of the sphere. But if the pendulum is removed from its angles, if the weight balance between the two shafts disappears, the robot's angle of rotation will change. This change is achieved when the pendulum closes to one of the two shafts, causing the gravity of the earth to adjust the robot so that the pendulum is closer to the ground, causing one of the shafts to be less than the time To the other shaft, the robot will eventually move in the direction the pendulum changes.

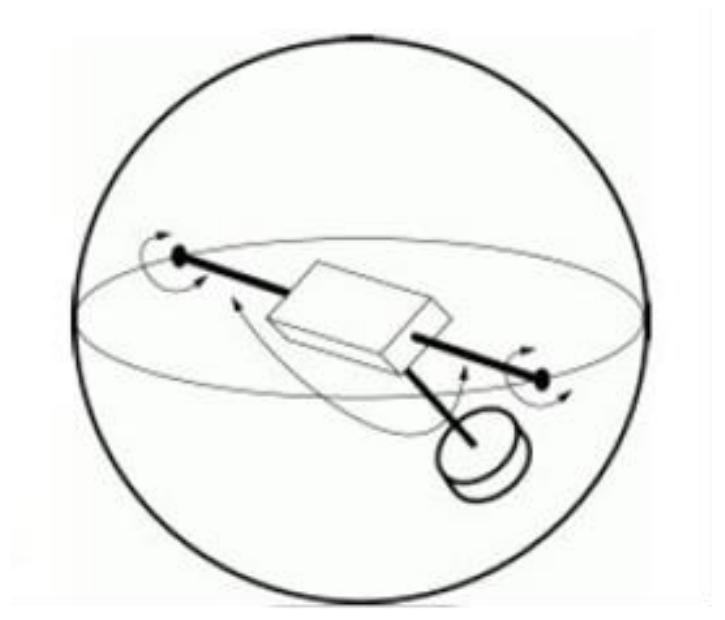

Figure. 3. Robot Motion Mechanism

\subsection{Electronic}

The electronic components and circuits of this project are divided into two parts:
A) An electronic module inside the robot that contains the following components.

- Rechargeable battery

- Voltage regulators 


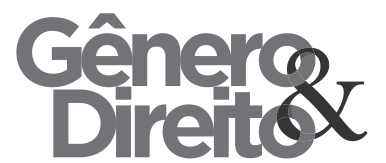

Periódico do Núcleo de Estudos e Pesquisas sobre Gênero e Direito

Centro de Ciências Jurídicas - Universidade Federal da Paraíba V. 8 - No 02 - Ano 2019 - Special Edition ISSN | 2179-7137 | http://periodicos.ufpb.br/ojs2/index.php/ged/index
- Switch gearboxes

- Electronic module for wireless communication (nRFL24L01P)

- IC controller (AT MEGA328)
- Accelerometer module and gyroscope (MPU9250)

- Compass module (GY 282)

- Geolocation GPS coordinate module

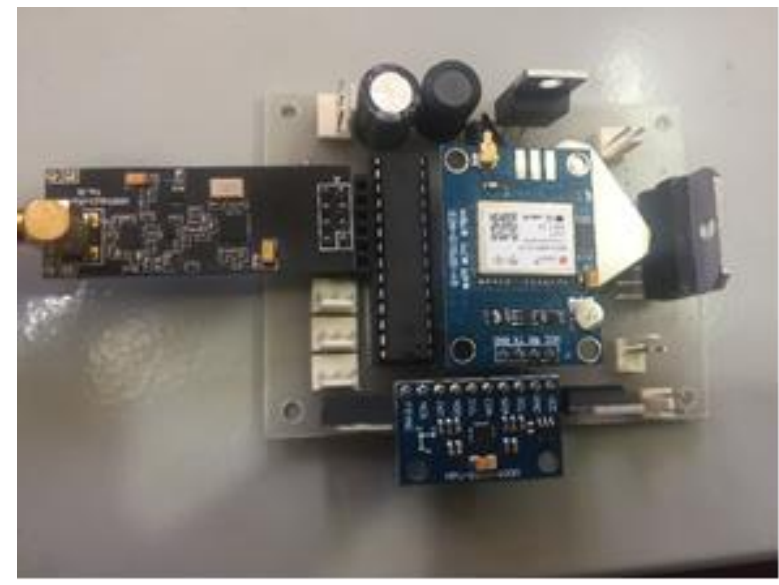

Figure. 4. The internal board of the robot

B) An electronic module attached to a computer that contains the following components.

- USB
- An electronic module for wireless communication (nRFL24L01P)

- Voltage regulator

- IC controller (AT MEGA8)

- USB converter to serial

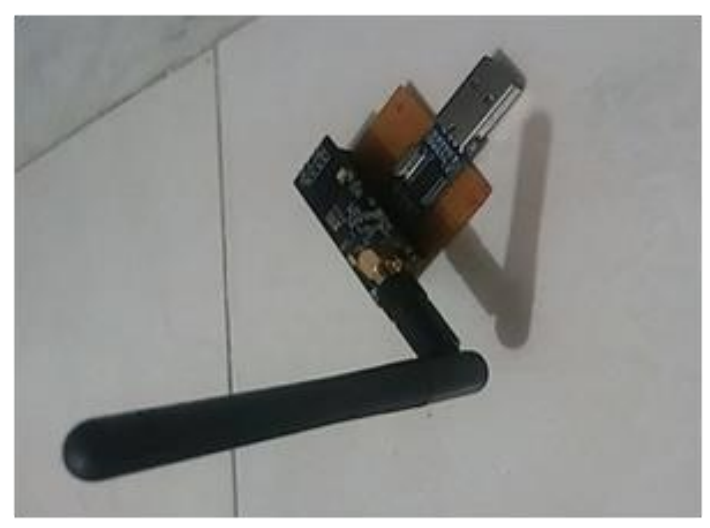

Figure. 5. The external board of the robot 


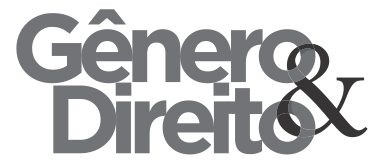

Periódico do Núcleo de Estudos e Pesquisas sobre Gênero e Direito

Centro de Ciências Jurídicas - Universidade Federal da Paraíba V. 8 - No 02 - Ano 2019 - Special Edition

ISSN | 2179-7137 | http://periodicos.ufpb.br/ojs2/index.php/ged/index
The following sections will be discussed and the methodology will be elaborated in detail.

\section{- $\quad$ Rechargeable battery}

The battery provides the energy needed to feed the electronic module and spherical robot engines, and the battery can be recharged and can be charged by the socket. The battery is positioned inside the globe, That the robot is equally weighted and It also plays the role of the pendulum inside the robot.

\section{- Voltage regulator}

Since each part of the robot, both motors and various parts of the electronic module each require different voltages for feeding, we use three regulators for feeding.
The first regulator (LF7805) converts a voltage of 12 volts to a voltage of 5 volts (stabilized), this $5 \mathrm{~V}$ voltage is used to feed the microcontroller, motor drivers and infra-red gauges, accelerometer and gyroscope and GPS modules.

The second regulator (LF33), which uses the voltage of the first regulator to feed its input,

The 3.3 volt fixed voltage is transmitted to the power supply to the wireless communication circuitry (nRF24L01).

The third regulator is the LM2576, which is used exclusively for servo motors.

12 volt batteries are used to start gearboxes.
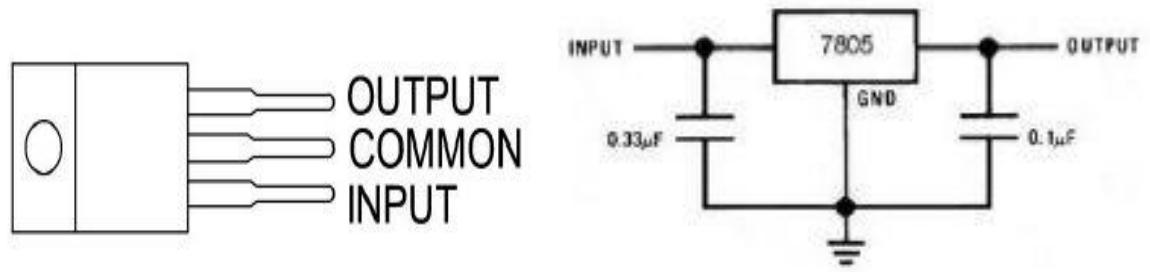

Figure. 6. Type I and II regulators 


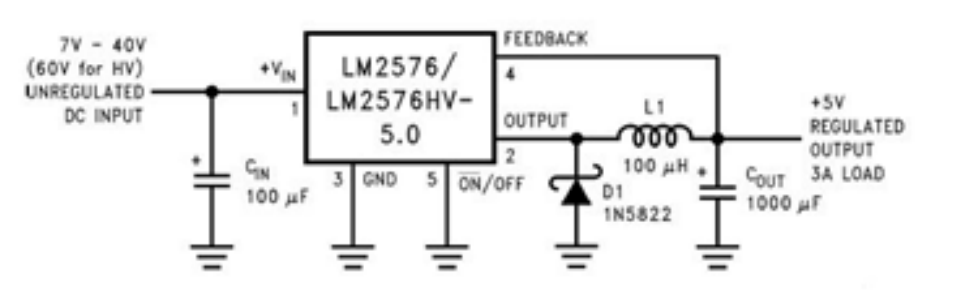

Figure. 7. LM2576

\section{- $\quad$ Switch gearboxes}

The driver (IC-L298) used in the electronics board of the robot, is used to setup and control two gearboxes and amplifies the signal transmitted by the microcontroller.

This driver uses two internal switches to run two engines. It has two inputs for each motor from the microcontroller and can set up two motors individually.

One of the characteristics of this driver is the permissible flow of one amperes for motors, the left and right turning of motors with pulse power input at PWM (speed control).

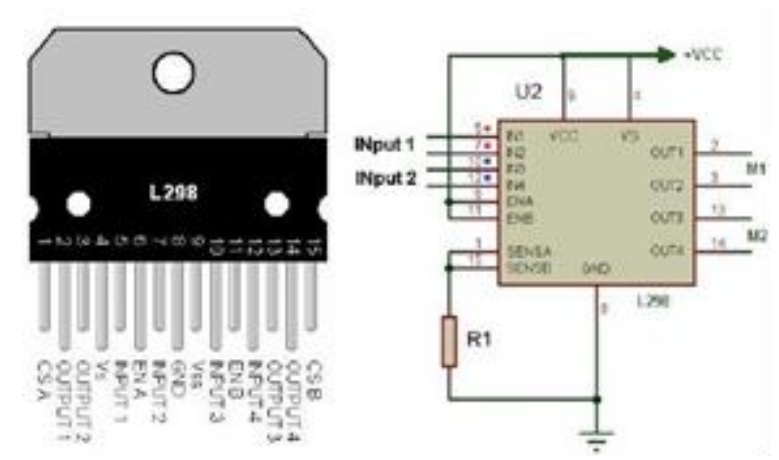

Figure. 8. L298 IC

- An electronic module for wireless communication

The masterpiece of this single chip 2.4GHz Transceiver module is brand + NRF24L01. Undoubtedly, this chip is one of the masterpieces of Nordic
Semiconductor. The high-end chip + NRF24L01 is a refined specification with more features and a higher data rate of the NRF2401 chip, introduced by NORDIC in early 2008. This chip is in fact an excellent module for sending and 
receiving error-free information. There was nothing in the HMTR modules, or even in RFMXXs, or that it had its own problems.

The modular communication module of this module is GFSK, the modularity used in BLUETOOTH technology and exclusively in the hands of large and advanced companies such as BLUETOOTH, NORDIC SEMICONDUCTOR and TEXAS INSTRUMENT. The communication frequency of this chip is $2.4 \mathrm{GHz}$. This feature brings a lot of features to us. Including a smaller antenna size, which can even be used as an antenna by the PCB itself. In addition, due to its high frequency, it easily passes through walls or other objects. And it will cause more wins. By comparing the frequency of RFM12 or HMTR with this chip, you see that the frequency of this module is at least equal to that. This chip works bilaterally, and in total only 2 of these chips are needed for communication.

The data chip has a maximum data rate of 2 megabits per second. Which can be used to transmit the same heavy audio data, or even video.

The + NRF24L01 chip is essentially designed for situations that require very low power consumption. In total, this chip requires just one microcontroller and a few other components, including multiple capacitors and inductors.

The communication protocol of this SPI chip can be managed and controlled by all protocols on all internal hardware and software features of the chip. The GFSK chip modulation is designed to match each chip with a specific frequency channel, so the percentage of error and chip impedance with each other or chips other than (+ NRFL24L01) is zero. The data rate of chip is configured at 2 megabits per second and has two different low-power modes that also makes it less consumable.

We can safely say that the + NRF24L01 + module, working on a 2.4 $\mathrm{GHz}$ frequency range, is really a very ideal module for similar prototypes. In addition to its perfect performance, the interior settings also offer more possibilities than imagination.

For example, due to some of the internal configurations in this project, all the necessary predictions have been made for the accuracy of received data, and the receiver module sends confirmation of receipt of the data to the sender module after receiving the data. 


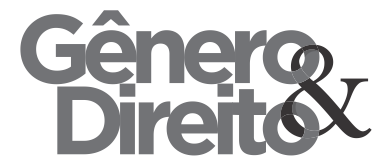

Periódico do Núcleo de Estudos e Pesquisas sobre Gênero e Direito

Centro de Ciências Jurídicas - Universidade Federal da Paraíba V. 8 - No 02 - Ano 2019 - Special Edition

ISSN | 2179-7137 | http://periodicos.ufpb.br/ojs2/index.php/ged/index
The transmitter module continues to receive data confirmation after receiving the bit. Otherwise, it stops the sending operation and resumes sending the data after it is stable.

If you notice a bit, this method of sending and receiving data is the same as Resume in Download Manager.

That is, you can stop sending data without writing a program at any stage, then send the data to the data, and all these operations are done directly to the hardware algorithms implemented inside this chip.

The chip + NRF24L01 is presented in the form of two modules, the first model has a microstrip antenna and a range of about 100 meters, but the second model is equipped with a PA amplifier and a LNA amplifier and an external antenna that operates at a frequency of $2.4 \mathrm{GHz}$. The antenna boost in the second model will increase the range to over a kilometer.

$$
\text { Compared to the }+ \text { nRF24L01 }
$$

module, a PA amplifier with a maximum output power of $+20 \mathrm{dBm}$ and a low noise amplifier class or LNA has been added to the NRF24L01P module, which ultimately increases the data transfer bandwidth and stability of the module's performance in industrial environments. . The product combines amplifiers for PA and LNA power, RF switches and intermediate pass filters for a two-way RF amplifier. In this study, the amplifier was used for this module.

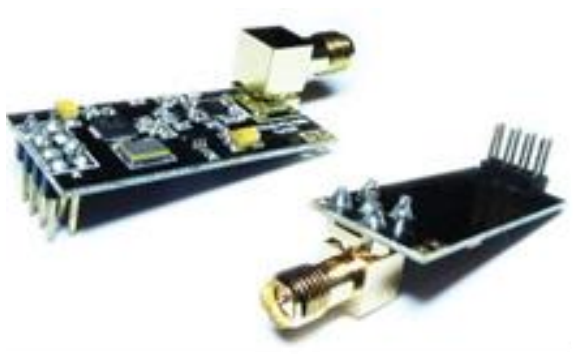

Figure. 9. Module + NRF24L01 


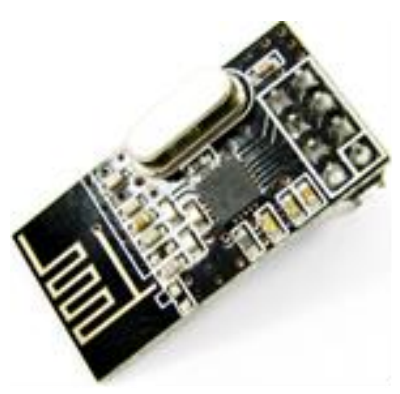

Figure. 10. Module NRF24L01P

The NRF24L01P module supports the SPI communication protocol. But there is a small problem, and that is, the module has a sixteen-bit SPI. While we use eight-bit SPI on most microcontrollers and in the AVR. Therefore, we need to solve this problem. In the following two methods are presented to solve this problem:

Method One: One way to solve this problem is to connect a base to the module instead of using the SSO 's select slave' in the SPI protocol and to work with the manual control of this base.

The second method is to use multiline programming code that can simulate the sixteen-bit SPI. In this method, the module information must be read simultaneously by sending the data to the module.
It should be noted that the second method has been used in this project. Information in 16-bit packages ( 2 bytes) is sent to the module. These two bytes contain the address of the registry and the data or settings. The address of each registry usually contains 8 bits of value or 4 bits of sent data value and the module will be notified at this address that the registry should be located in which data.

- $\quad$ AT-MEGA328

Microcontroller

The main controller and coordinator of electronic components in the construction of this project is the ATMEGA328, which is manufactured by Atmel Company. 


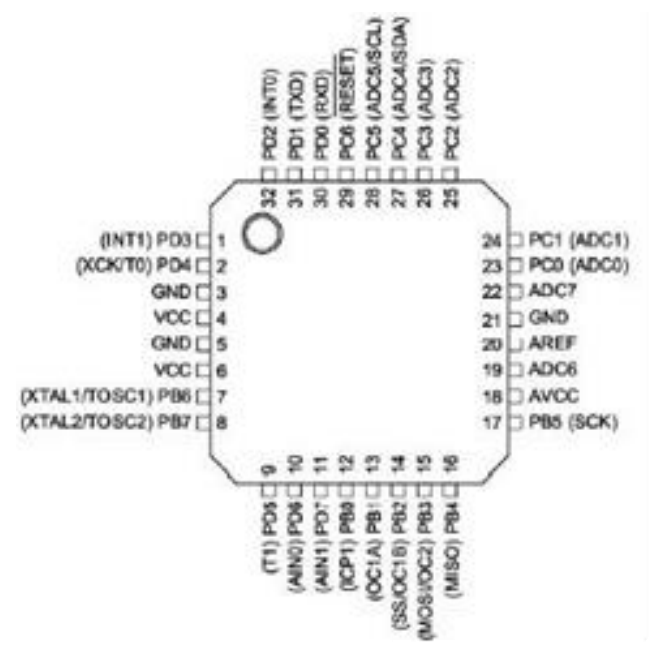

Figure. 11. AT-MEGA328

This microcontroller is programmable in $\mathrm{C}++$. As seen in the PCB, no external crystal is used for the IC clock, and instead of an $8 \mathrm{MHz}$ IC crystal is used.

\section{- Accelerometer and gyroscope module}

The MPU9250 is a combination of two accelerometer and gyroscope functions. This module provides almost all the requirements for the creation of an internal measurement system with 9 degrees of freedom and 9 axes, with a high precision and advanced internal digital motion processing (DMP) system of the features of this module. The module consists of two distinct chips, each of which has been briefly described, depending on the $\mathrm{I} 2 \mathrm{C}$ interface, it can easily be set up with educational boards or any other microcontroller. The module includes a three-axis gyro sensor with a precision of 16 bits and a range of 2000 degrees per second, a three-axis accelerometer with a precision of 16 bits and a measuring range of up to $16 \mathrm{~g}$, a 3 axis compass sensor with a precision of 13 bits, also has a FIFO buffer With a capacity of 1024 bytes and a temperature sensor with a range of measurements of -40 to $+85^{\circ} \mathrm{C}$. 


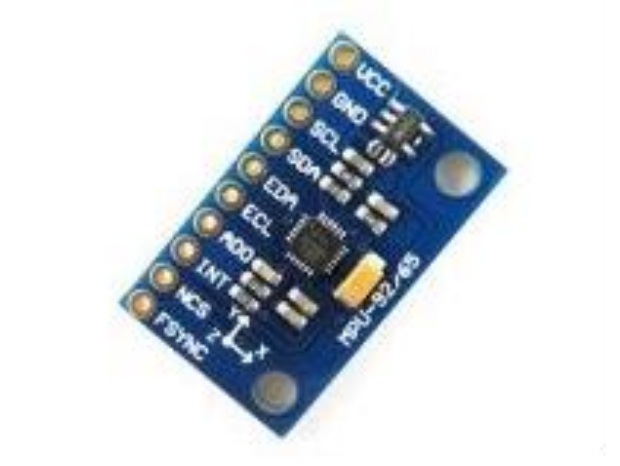

Figure. 12 . MPU9250

\section{- GPS}

The GPS module is able to calculate spatial, temporal, velocity and acceleration data at any moment and provide the user with an output. These modules perform calculations based on the delayed reception of signals from satellites. The GPS module can tell the sunrise and sunset times in your position. The GPS can calculate the remaining time to reach the desired destination according to the speed of the object. Also, the average speed, the highest speed, the average ascending and descending path, the vertical speed, the location of the area in terms of hunting and fishing and hunting in any part of the world, the calculation of the area of an unknown point, and returning the object from the path that passed it can also do.

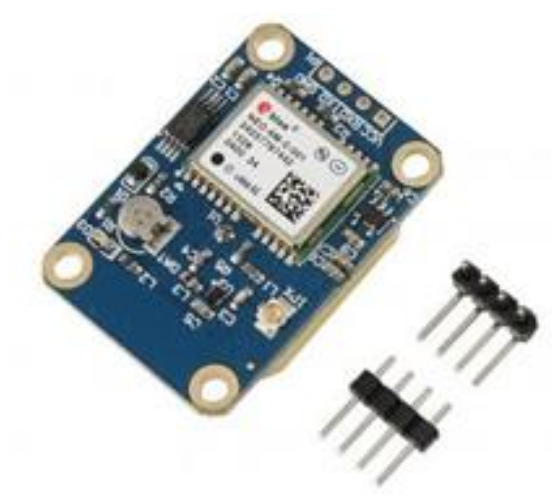

Figure. 13. GPS

The NEO-6 series modules are from the family of U-blox GPS receivers that have been designed and manufactured based on the U-blox's 
powerful locator. These GPS receivers are so flexible and powerful that they offer many features in small dimensions to the user.

The orbital architecture of this module and its features in energy consumption and internal memory, make the NEO-6M suitable for use in systems with battery power and portable equipment. The design of the NEO-6M module circuits is such that the module's startup time for the first trusted output, or TTFF, has been reduced to less than one second. The satellite search engine in this module, with two million Correlator, is able to find instantaneous satellitebased satellites with parallel-time and frequency searches. The design and technology used in this module can eliminate the effects of jamming or distortion sources of GPS signals. In general, the NEO-6 series of GPS receivers has a good performance in receiving navigation signals, even in high noise environments.

\section{- Infrared distance meter sensor}

The GP2Y0A21YK0F distance meter module is an IR distance meter module that uses infrared light for distances. The output of the module is of analog voltage type, which can be easily distorted by microprocessor ADC channel. This module can be used in the industry, especially in robotics.

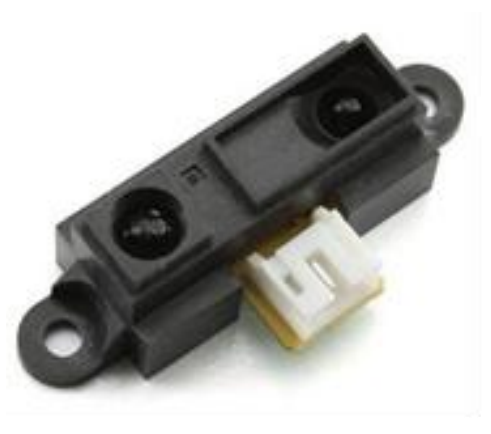

Figure. 14. GP2Y0A02YK

\subsection{Computer}

In this section, we will examine the prototype drawings in the proteus and describe how the robot works.
The spherical robot made up of two single-board spheres in the sphere and the other is connected to the laptop, the speed, position and angle information transmitted by the wireless connection from the robot to the laptop. 


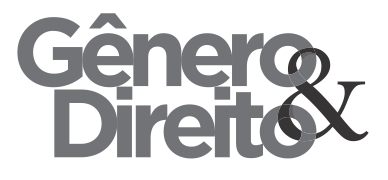

Periódico do Núcleo de Estudos e Pesquisas sobre Gênero e Direito

Centro de Ciências Jurídicas - Universidade Federal da Paraíba V. 8 - No 02 - Ano 2019 - Special Edition
In automatic mode, the scheduled system operates in such a way that, by comparing the current position and the final position determined on the map by a SMC controller, calculates the angle, speed, and length of the robot's motion, and ultimately, through wireless
46

communication The robot orders the move. This process continues until the robot reaches the designated destination on the map. In manual control mode, the angle, speed and length of movement by the person is given directly to the robot through the strips embedded on the map.

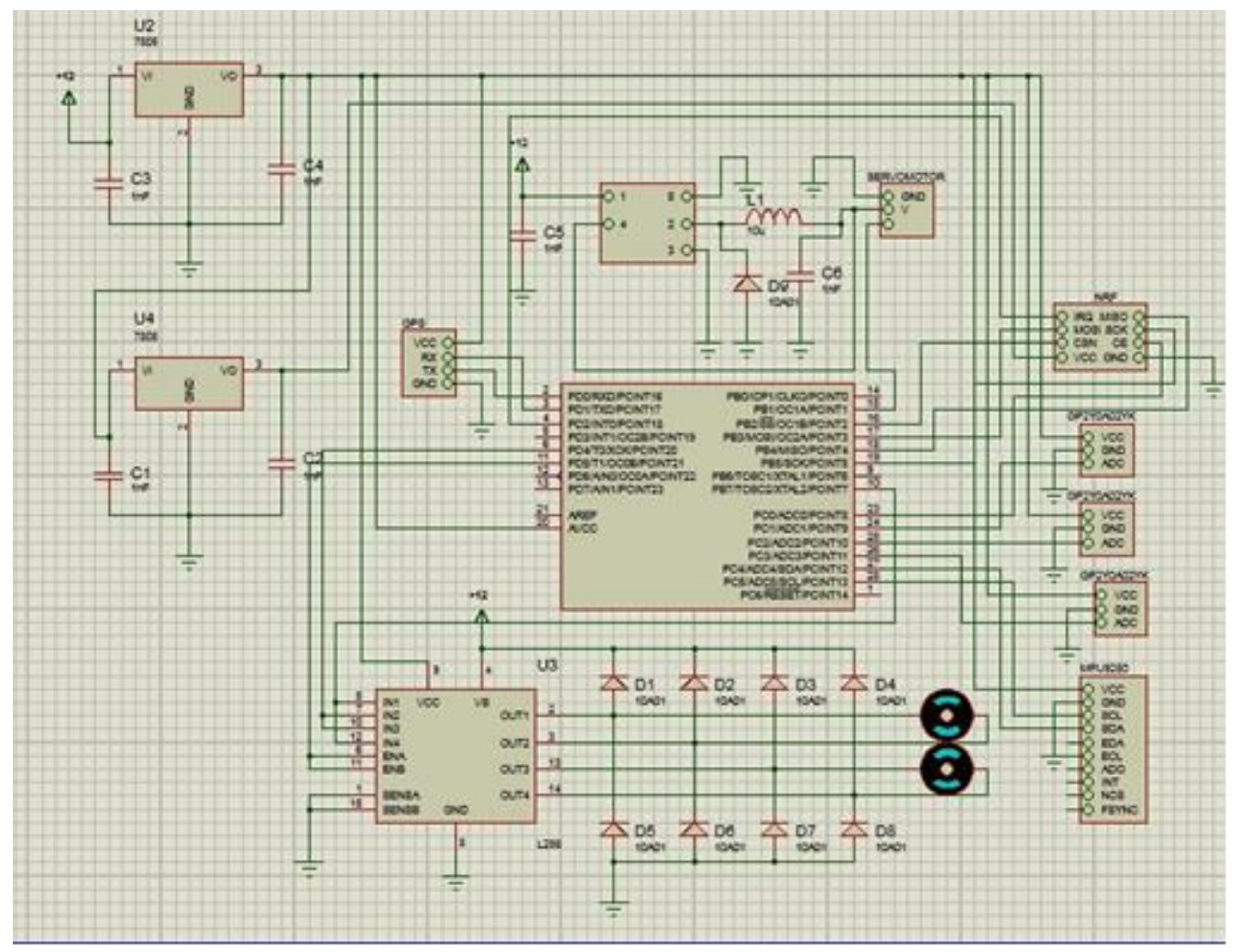

Figure. 15. Schematic view of receiver board 


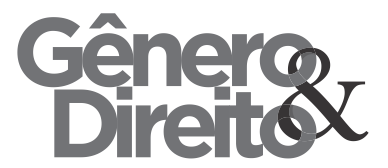

Periódico do Núcleo de Estudos e Pesquisas sobre Gênero e Direito

Centro de Ciências Jurídicas - Universidade Federal da Paraíba V. 8 - No 02 - Ano 2019 - Special Edition ISSN | 2179-7137 | http://periodicos.ufpb.br/ojs2/index.php/ged/index

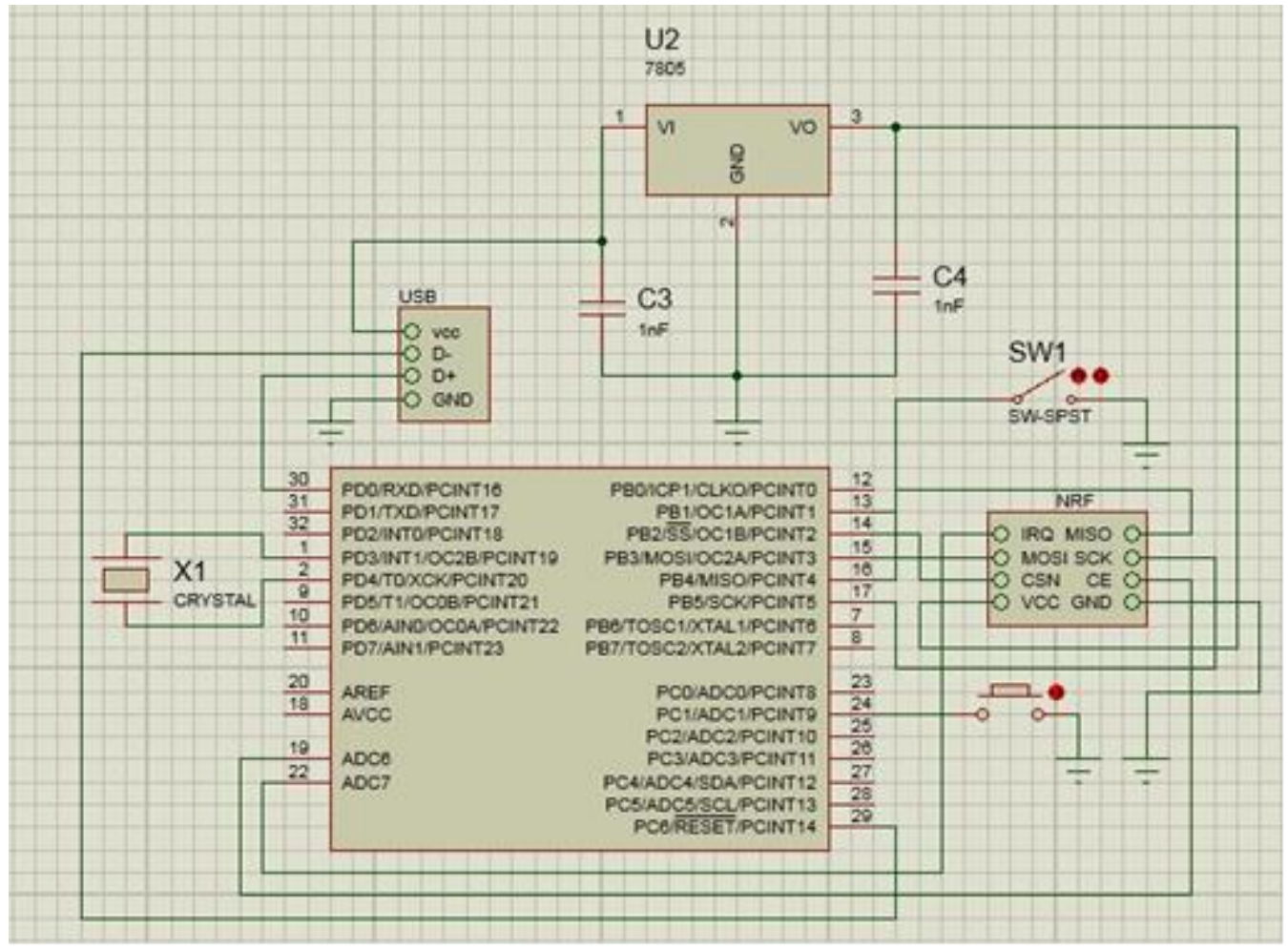

Figure. 16. Schematic view of the transmitter board

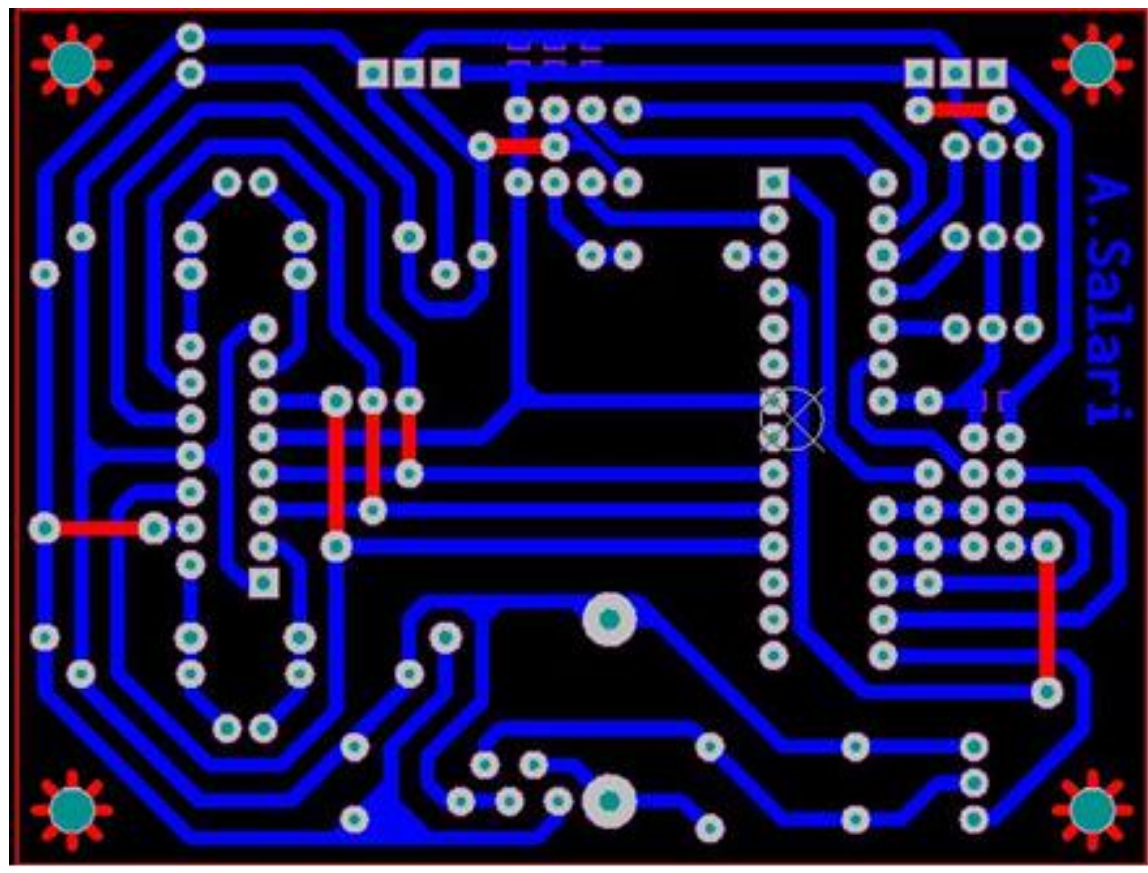

Figure. 17. PCB 


\section{Gênerg Direital}

\subsection{1 sliding mode control}

A SMC is a type of Variable Structure Control (VSC). It uses a high speed switching control law to force the state trajectories to follow a specified, user defined surface in the states space and to maintain the state trajectories on this surface. The control law for a SMC consists of two parts; a corrective control part $U_{c}(t)$ and an equivalent control part $U_{e q}(t)$. The corrective control function is to compensate any variations of the state trajectories from the sliding surface in order to reach it. The equivalent control on the other hand, makes sure the time derivative of the surface is

maintained to zero, so that the state trajectories would stay on the sliding surface.

$U(t)=U_{c}(t)+U_{e q}(t)$

In this section, we want to control the position of the robot by controlling the inputs. Two outputs of the robot can be controlled because this system has two inputs. $X, Y$ that will be considered outputs in order to control the position of spherical robot. Besides, the angular velocities are considered as inputs. Kinematic equations are rewritten as follows.

$\dot{P}=J N$

$P=\left[\begin{array}{l}X \\ Y\end{array}\right], \quad J=\left[\begin{array}{cc}-\rho \cos \beta \sin \xi & \rho \cos \xi \\ \rho \cos \beta \cos \xi & \rho \sin \xi\end{array}\right], \quad N=\left[\begin{array}{c}\dot{\alpha} \\ \dot{\beta}\end{array}\right]$

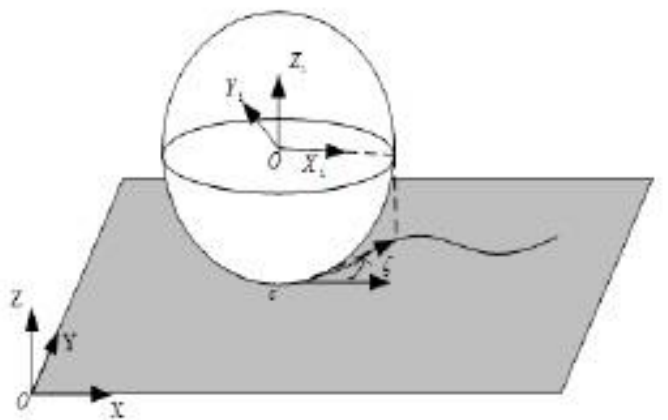

Figure. 18. The holonomy angle in the spherical robot 
where, $\rho, \beta$ and $\alpha$ are position coordinates. The contact path on the plane is determined by $\mathrm{c}=(\mathrm{x}, \mathrm{y})$ in the xyz-coordinates connected to the plane In this section will be to design a control sliding surface, first, a sliding surface vector will be defined as follows.

$e_{P}=P-P_{d}$

$s_{P}=\lambda_{1}\left(e_{P}\right)+\left(\dot{e}_{P}\right)$

$\dot{s}_{P}=\lambda_{1}\left(\dot{e}_{P}\right)+\left(\ddot{e}_{P}\right)=0$

In (3), $p_{d}$ is the centre of the robot in desirable position.

According to formula $1, \mathrm{u}$ is composed of two parts where $u_{e q P}$ includes parameters that are known and can be deleted and $u_{c P}$ uses to remove noise and uncertainty.

$u_{e q P}=J^{-1}\left(\dot{p}_{d}+\gamma s\right)$

To design $u_{c z}$ a Lyapunov function $V$ is defined. This function must be positivedefinite.

$V=\frac{1}{2} s^{T} M s>0 \quad M=\left[\begin{array}{cc}1 & 1 / 2 \\ 1 / 2 & 1\end{array}\right]$

The derivative of the function $V$ must be negative-definite.

$\dot{V}=s \dot{s}<0$

In order to satisfy equation $6 u_{c z}$ should be:

$u_{c P}=-\eta M^{-1} \operatorname{sign}\left(s_{P}\right)$

Where $\mathrm{s}$ is defined by (3).

The reaching condition is given by the following inequality $\dot{V}<0$. Therefore, to satisfy it:

$\eta>0$ for all $t>0$

Thus, the complete controller equation is

$u=u_{e q P}+u_{c P}$

\subsection{2 simulation result}

This section provides the simulation results through MATLAB, to verify the efficiency of sliding mode controllers. Considering the robot rolls without slipping on a plane. Initial 


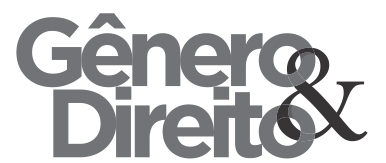

Periódico do Núcleo de Estudos e Pesquisas sobre Gênero e Direito

Centro de Ciências Jurídicas - Universidade Federal da Paraíba V. 8 - No 02 - Ano 2019 - Special Edition

ISSN | 2179-7137 | http://periodicos.ufpb.br/ojs2/index.php/ged/index

conditions for simulation are presented

as follows.

$P_{d}=\left[\begin{array}{c}X_{d} \\ Y_{d}\end{array}\right]=\left[\begin{array}{c}2 \sin (2 t) \\ \sin (t)\end{array}\right]$

$P\left(t_{0}\right)=\left[\begin{array}{l}X \\ Y \\ \Psi\end{array}\right]_{t_{0}}=\left[\begin{array}{c}3 / 4 \\ -3 / 4 \\ 0\end{array}\right]$

Radius of the robot is assumed to be $\rho=0.2$. The simulated of sliding mode control are as follows. Desired trajectory and robot motion path are given in Figure.20. Control inputs for desired path from initial point to final state will be as follows:
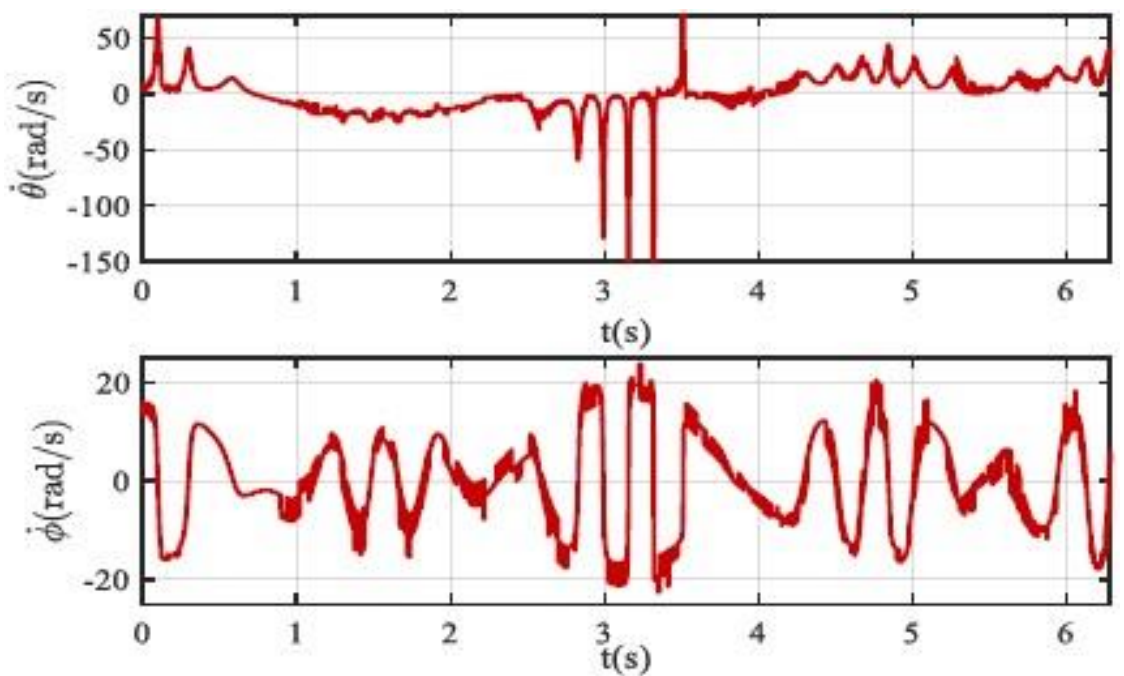

Figure. 19. Robot inputs 


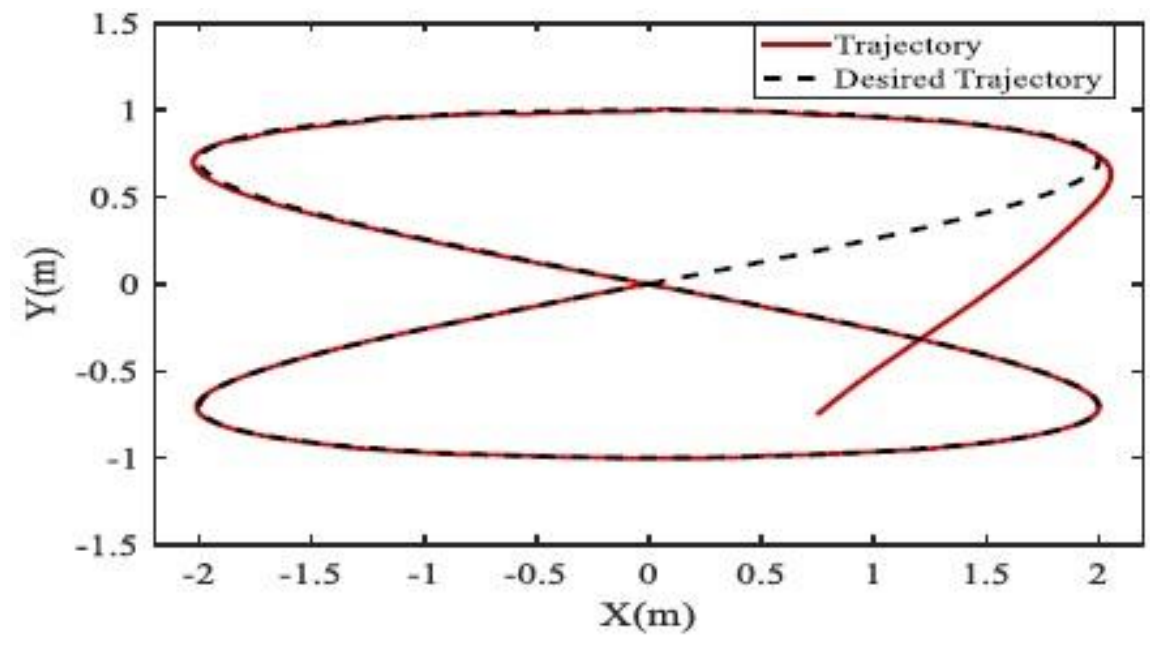

Figure. 20. Robot trajectory along $x-y$ plane

\section{CONCLUSION}

Today robots and robotics are in progress which meets more human needs. Various types of robots are made of humane and industrial, but among them robberies are more valuable to facilitate more difficult conditions for humans. Spherical robot can work in all difficult conditions that humans can not even have the ability to be there. Including the presence of gas pipelines to detect leaks, the presence of war zones for carrying weapons, space travel and filming, crossing high-rise and hard-line agricultural routes to diagnose product and pest disease, even at the borders $\mathrm{A}$ sea to detect mines and prevent foreign forces from invading.

These are not all spherical robot features. Remote control using wireless communication has added to its advantages particularly, the ability to manually control is also added. The high-range wireless connection allows the robot to follow a large distance from the map and go to the location specified by the operator.

The robust feature that makes it distinct from other robots is its spherical shape. Internal space allows it to add parts or carry additional objects inside it, and it increases the capabilities of the spherical robot and the ability to develop hardware.

\section{REFERENCES}


Journal of aerospace mechanics (Dynamics, vibration and control), Vol 7, no.1, 1390 construction, planning and setting up of spherical robot, Alireza Ajdari Faramani.

Yaxin Li, shuxiang Guo, Yu Wang, Design and characteristics evaluation of a novel spherical underwater robot, robotics and Autonomous systems, volume 94, August 2017

Maoxun Li, shuxiang Guo, Hideyuki HirataT Hidenori Ishihara, Design and performance evaluation of an amphibious spherical robot, robotics and Autonomous Systems, volume 64, Februay 2015

Wei-His chen, Ching-pei chen, Jiashiuan Tsai, Jackie Yang, Pei Chun lin, Design and implementation of a balldriven omnidirectional spherical robot, IFAC-PapersOnLine, Volume 49,Issue 18,2016

S. Bhattacharya ; S.K. Agrawal , Design, experiments and motion planning of a spherical rolling robot, Proceedings 2000 ICRA. Millennium Conference. IEEE International Conference on Robotics and
Automation. Symposia Proceedings

(Cat. No.00CH37065) , Volume: 2 ,Year: 2000

Chia-Wen Wu ; Zhong-Wei Qiu ; YenHsiang Wang ; Po-Hsiang Hsu ; ChiKuang Hwang, Modeling of a spherical robot driven by Omni wheels , 2011 International Conference on Machine Learning and Cybernetics, Volume: 3 ,Year: 2011

Murniwati Anwar ; Lukman Hakim Omar; Yusof Mohd Ekhsan ; Ruwaida Ramli ; Nurzulaikhah Nadzri ; Fazil Ahmad ; Muhammad Hazman Shafii, Conceptual of spherical robot , 2014 11th International Conference on Ubiquitous Robots and Ambient Intelligence (URAI) ,Year: 2014

Kang Hou ; Hanxu Sun ; Qingxuan Jia ; Yanheng Zhang, The Design of the Spherical Robot Autonomous GPSBased Positioning and Navigation System , 2010 International Conference on Intelligent System Design and Engineering Application ,Volume:2 , Year: 2010

Zhe Wang ; Shuxiang Guo ; Liwei Shi ; Shaowu Pan; Yanlin $\mathrm{He}$, The 


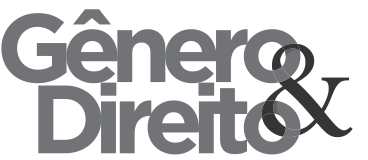

Periódico do Núcleo de Estudos e Pesquisas sobre Gênero e Direito

Centro de Ciências Jurídicas - Universidade Federal da Paraíba V. 8 - No 02 - Ano 2019 - Special Edition ISSN | 2179-7137 | http://periodicos.ufpb.br/ojs2/index.php/ged/index application of PID control in motion control of the spherical amphibious robot , 2014 IEEE International Conference on Mechatronics and Automation , Year: 2014

Joshi, Vrunda A., and Ravi N. Banavar. "Motion analysis of a spherical mobile robot." Robotica 27.03 (2009): 343-353.

Joshi, Vrunda A., Ravi N. Banavar, and Rohit Hippalgaonkar. "Design and analysis of a spherical mobile robot." Mechanism and Machine Theory 45.2 (2010): 130-136.

Kamaldar, M., et al. "A control synthesis for reducing lateral oscillations of a spherical robot." Mechatronics (ICM), 2011 IEEE International Conference on. IEEE, 2011.

Roozegar, M., M. J. Mahjoob, and M. Jahromi. "DP-based path planning of a spherical mobile robot in an environment with obstacles." Journal of the Franklin Institute 351.10 (2014): 4923-4938.

Roozegar, M., M. J. Mahjoob, and A. Shafiekhani. "Using Dynamic Programming for Path Planning of a Spherical Mobile Robot."
Marigo, Alessia, and Antonio Bicchi. "A local-local planning algorithm for rolling objects." Robotics and Automation, 2002. Proceedings. ICRA'02 